\author{
Rohani Omar \\ Elizabeth L. Sampson \\ Clement T. Loy \\ Catherine J. Mummery \\ Nick C. Fox \\ Martin N. Rossor \\ Jason D. Warren
}

\section{Delusions in frontotemporal lobar degeneration}

Received: 27 May 2008

Received in revised form:

17 September 2008

Accepted: 17 October 2008

Published online: 9 April 2009

Dr. J. D. Warren $(\varangle) \cdot$ R. Omar

C. J. Mummery - N. C. Fox · M. N. Rossor

Dementia Research Centre

Institute of Neurology

University College London

Queen Square

London, WC1N 3BG, UK

E-Mail: jwarren@drc.ion.ucl.ac.uk

E. L. Sampson

Dept. of Mental Health Science

RFUCMS, UCL, London

C. T. Loy

Prince of Wales Medical Research Institute, Randwick

Sydney, Australia

\author{
C. J. Mummery · N. C. Fox · M. N. Rossor • \\ J. D. Warren \\ National Hospital for Neurology \\ and Neurosurgery \\ Queen Square \\ London, UK
}

Abstract We assessed the significance and nature of delusions in frontotemporal lobar degeneration (FTLD), an important cause of young-onset dementia with prominent neuropsychiatric features that remain incompletely characterised. The case notes of all patients meeting diagnostic criteria for FTLD attending a tertiary level cognitive disorders clinic over a three year period were retrospectively reviewed and eight patients with a history of delusions were identified. All patients underwent de- tailed clinical and neuropsychological evaluation and brain MRI. The diagnosis was confirmed pathologically in two cases. The estimated prevalence of delusions was $14 \%$. Delusions were an early, prominent and persistent feature. They were phenomenologically diverse; however paranoid and somatic delusions were prominent. Behavioural variant FTLD was the most frequently associated clinical subtype and cerebral atrophy was bilateral or predominantly right-sided in most cases. We conclude that delusions may be a clinical issue in FTLD, and this should be explored further in future work.

Key words delusions . frontotemporal lobar degeneration - Pick's disease · dementia

\section{Introduction}

Frontotemporal lobar degeneration (FTLD) is a group of degenerative conditions characterized by progressive focal frontal and/or temporal lobe atrophy that collectively constitute a common cause of young-onset dementia. Three major FTLD syndromes are recognized: behavioural variant (bvFTLD), presenting with predominant behavioural and executive problems; temporal variant FTLD (tvFTLD) presenting as impaired semantic memory for verbal material (semantic dementia, SD) or nonverbal material such as faces (progressive prosopagnosia); and progressive non-fluent aphasia (PNFA) presenting with predominant speech production diffi- culties $[1,2]$. Group studies have delineated characteristic patterns of brain atrophy on magnetic resonance imaging (MRI) [3-5]. The clinical and neuroanatomical phenotypes are underpinned by a heterogeneous spectrum of histopathological abnormalities and disease associations (in particular, motor neurone disease) [6]. Deposition of abnormal cellular inclusions containing proteins tau or ubiquitin is a key pathological feature of diseases in the FTLD spectrum and mutations in the genes coding the relevant proteins account for a relatively high proportion of cases. However the pathophysiological mechanisms whereby these molecular signatures translate into regional brain damage and clinical phenomenology have not been defined.

Neuropsychiatric disturbances occur in all subtypes 
of FTLD, particularly bvFTLD $[7,8]$. The spectrum of neuropsychiatric phenomena in FTLD is wide and the true status of behavioural and cognitive alterations may be difficult to establish. Diagnosis is often difficult and a primary psychiatric illness may be suspected. While certain behavioural manifestations of FTLD such as abulia or rituals may suggest primary psychiatric illness [9], diagnostic confusion is more likely to arise in patients with FTLD who have prominent positive psychotic symptoms (delusions and hallucinations). Psychotic symptoms are generally regarded as an uncommon feature of FTLD [10], and more often associated with other neurodegenerative conditions such as dementia with Lewy bodies (DLB) and Alzheimer's disease (AD) [11, 12]. Delusions are especially pertinent to this issue, since (in contrast to hallucinations) they are less widely recognized as harbingers of structural brain disease and more likely to lead to psychiatric misdiagnosis. Aside from their implications for clinical diagnosis, delusions in FTLD are of considerable neurobiological interest due to the potential insights they hold into the brain mechanisms that link information about external reality with internal representations of the world. Such mechanisms are likely to involve neural networks in the frontal and temporal lobes that are particularly vulnerable in FTLD [13].

Here we describe in detail a series of patients with FTLD who developed delusions. Our primary aim was to assess the frequency and nature of delusions in FTLD. A secondary aim was to explore any associations with the clinical and/or neuroanatomical subtype of FTLD.

\section{Method}

The case notes of all patients meeting consensus clinical criteria for FTLD $[1,2]$ referred to a tertiary level cognitive disorders clinic during a three year period were retrospectively reviewed to identify patients describing delusions. We used the DSM-IV definition of a delusion [14] as 'a false belief based on an incorrect inference about external reality that is firmly sustained despite what almost everyone else believes and despite what constitutes incontrovertible and obvious proof or evidence to the contrary. The belief is not one ordinarily accepted by other members of the person's culture or subculture.' Delusions were assessed using a standard proforma based on the clinical information, including the time of onset in relation to other symptoms, any previous psychiatric history, whether associated hallucinations were present (and their modality), and the phenomenological content of the delusions. The clinical subtype, neuropsychological and neuroimaging findings, and histopathological findings (where available) were also recorded in each case. Eight cases with delusions were ascertained from 56 patients with a clinical diagnosis of FTLD referred to the clinic during this period. All patients had detailed clinical and neuropsychological assessments and supportive brain magnetic resonance imaging (MRI) and/or pathological findings (reviewed with an experienced neuroradiologist and neuropathologist). Data for all cases are summarized in Table 1. Representative brain images are shown in Fig. 1. Informed consent was obtained from subjects and carers to use the data in this study which was performed with the approval of the local institutional ethics committee and in accord with Declaration of Helsinki guidelines.

\section{Case descriptions}

Case 1

A 65 year old female academic presented with progressive personality, behavioural and cognitive changes over two years. She abandoned reading and other hobbies, and her demeanour became fatuous and childlike. She preferred children's television programmes and would
Fig. 1 Representative coronal T1-weighted brain MR images from patients in this series, to illustrate the range of anatomical disease patterns ( $L$ left side of brain): a) predominantly right temporal lobe atrophy (Case 7); b) predominantly left temporal lobe atrophy (Case 8); c) predominantly right frontotemporal atrophy (Case 6); d) bi-frontotemporal atrophy, more marked on the right (Case 5)
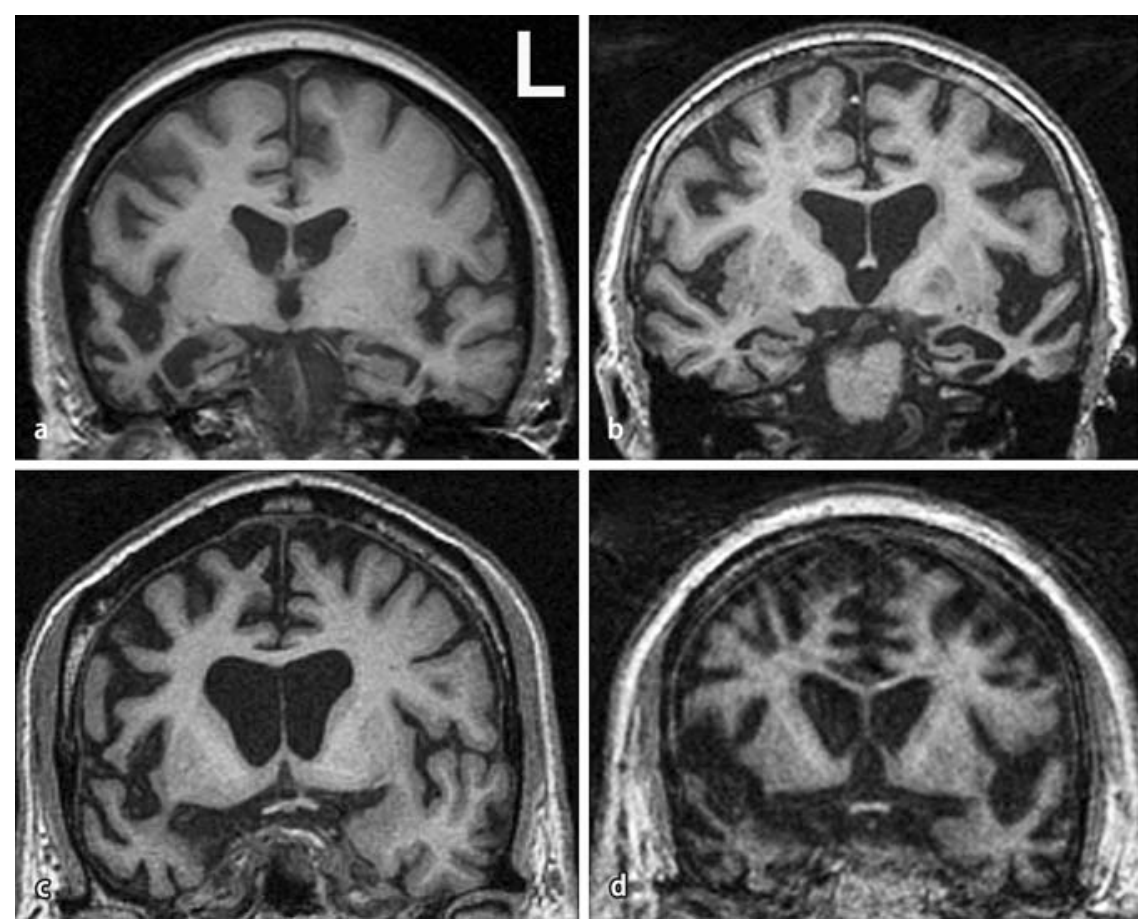


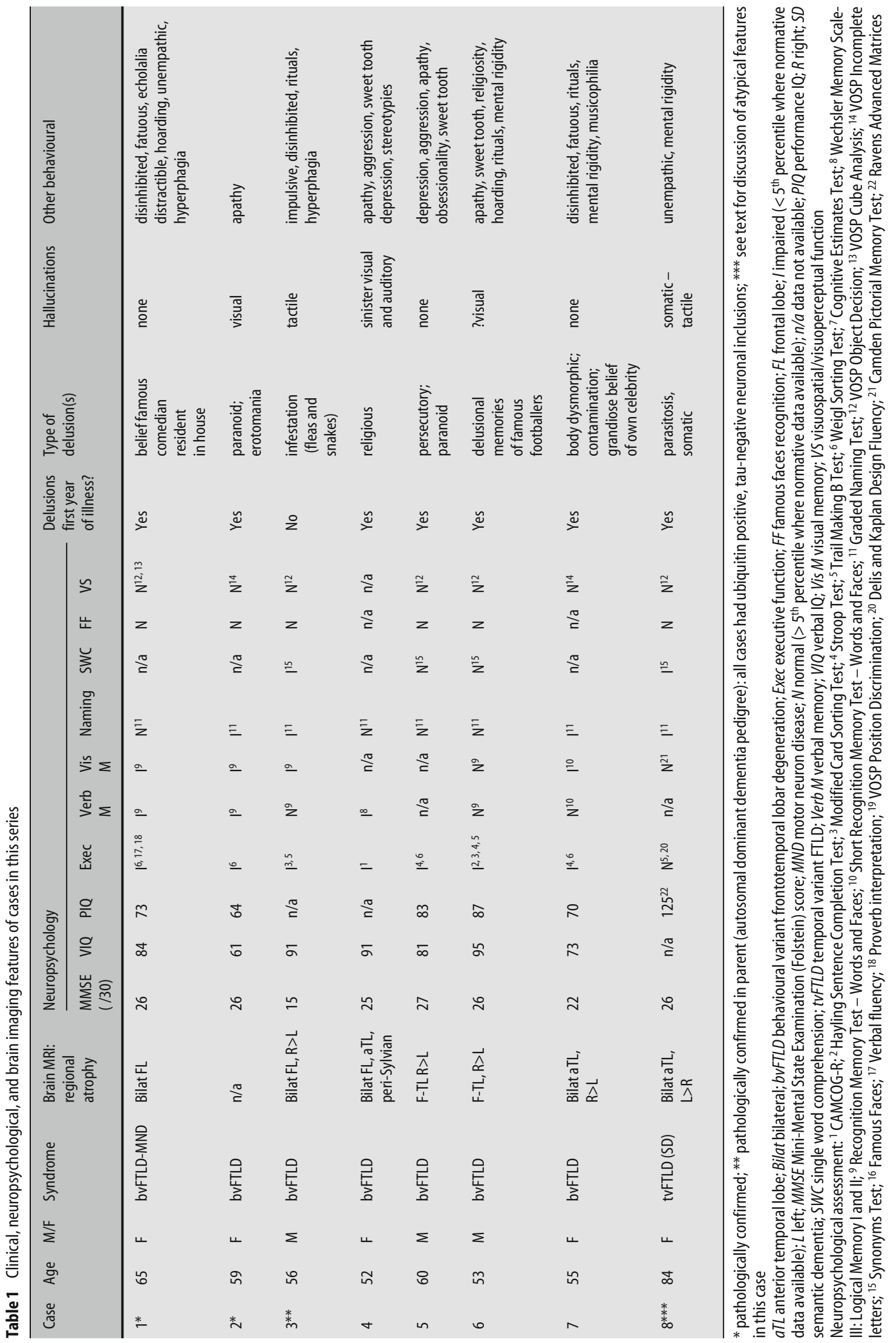


switch channels continually. She became less affectionate and empathic toward others, and would make embarrassing remarks in public and laugh inappropriately. She hoarded magazines and ate voraciously, with a preference for sweet foods, and required prompting to change her clothes. Delusions emerged within the first year of the illness. She insisted that a well-known British comedian and his family had moved into her house and she would prepare meals for them. She also believed there was a black panther living in the garden. She inferred their presence from sounds which she attributed to them, and could not be persuaded that these beliefs were false.

The past medical history included idiopathic thrombocytopenia, managed with oral prednisolone and splenectomy; she had received oral corticosteroid therapy for six years prior to the onset of behavioural symptoms, which began several months post- splenectomy while she was taking a tapered dose of prednisolone $(15 \mathrm{mg}$ alternate daily). There was no history of psychiatric illness, and no family history of dementia.

On initial examination she was impulsive, jocular and disinhibited. There was impaired attention, profound executive deficits and echolalia. There was wasting of hand and foot muscles with fasciculations of the left quadriceps, though no limb or bulbar weakness. Electromyography confirmed widespread denervation of upper and lower limbs consistent with anterior horn cell disease. Over the next four months she became profoundly echolalic and echopraxic, with ideational apraxia for limb and orofacial movements. The general neurological examination now revealed widespread fasciculations and generalised muscle wasting, and a left extensor plantar response.

The patient died following development of acute respiratory failure. Post mortem findings were those of frontotemporal dementia with motor neuron disease (FTD-MND).

\section{Case 2}

A 59 year old woman presented with a two-year history of paranoid beliefs that people were hiding in bushes outside her house and trying to break in. She insisted that she could see them and described their appearance as 'Rastafarian'. She also maintained that she was having an affair with a man whom her family confirmed did not exist, and that if she dialed 3 on the telephone she would get a direct line to him. She could not be persuaded that these beliefs were untrue. She became increasingly unmotivated, spending much of the day watching television and requiring prompting with activities of daily living. Her conversation became very limited.

She was initially given a diagnosis of paraphrenia and prescribed a combination of antipsychotics and antidepressants, with no improvement. She became profoundly apathetic with daytime somnolence. The past medical history was unremarkable. There was no previous psychiatric history and no known family history of dementia.

On examination, she exhibited flattened affect, with limited eye contact. Spontaneous speech was reduced and aprosodic. Concentration and memory for recent events were impaired. The general neurological examination was normal.

The patient died three years after symptom onset. Post mortem histopathological examination revealed ubiquitin-positive, tau and $\alpha$-synuclein-negative abnormal neurites and intraneuronal cytoplasmic inclusions.

\section{Case 3}

A 56 year old male builder presented with progressive cognitive and behavioural decline over four years. He took medical retirement on account of erratic work performance, he lost his driving licence due to recklessness, and he exhibited odd behaviors such as digging a canal in his garden. He became disinhibited and fatuous and engaged in compulsive hoarding and rituals. He developed hyperphagia and sweet tooth. There were no features of a major mood disorder. He developed word-finding difficulties with reduced spontaneous speech, became more forgetful and distractible, and had difficulty dressing. Three years into the illness, he was preoccupied with un- usual somatic sensations, for example complaining of generalised pruritus which he attributed to snakes or fleas crawling over his skin. This was an unshakable belief which, when challenged, he rationalised as in keeping with the behaviour of such creatures, and led him to shower so frequently that his skin became excoriated. He described the 'carpet snakes' in detail, although he had never seen them. He also described a sense of depersonalisation and feeling "out of his body". The past medical history included hypercholesterolemia, managed with atorvastatin. There was a strong autosomal dominant family history of dementia with frontotemporal features and his mother had been found to have ubiquitin-positive, tau-negative intraneuronal inclusions at post-mortem examination.

On examination, he was disinhibited, impulsive and perseverative. There was facial impassivity, impersistence of eye closure and tongue protrusion, a brisk jaw jerk and pout reflex. The general neurological examination was otherwise unremarkable.

\section{Case 4}

A 52 year old female shoe-shop assistant had developed progressive behavioural change over a period of 7 years. She initially described hearing a male voice she believed to be Satan speaking to her. The voice would order her to perform unpleasant, sinister acts such as harming her daughter or putting a hot iron to her own face. She believed that particular houses and places were associated with an evil presence, and on one occasion could not be persuaded by her family to enter an apartment on this account. She later developed unpleasant visual hallucinations of monsters. Work colleagues reported she was making mistakes with shoe sizes and acting inappropriately towards customers. Subsequently she became increasingly restless and distractible and would perform meaningless stereotypical actions. She took up smoking, developed a sweet tooth and exhibited anti-social behaviours such as stealing. She was placed in a long-term psychiatric assessment ward due to her profound behavioural difficulties. Trials of antidepressant and antipsychotic medication produced no benefit. She had no difficulties with topographical orientation or with recognizing familiar faces. There was no significant previous medical or psychiatric history. There was no family history of neurological illness, however a number of family members were described as having difficult personalities with drug abuse and social problems.

On examination, she was agitated. Cognitive estimates were abnormal and on a test of similarities, she provided verbose and concrete responses. The general neurological examination was unremarkable.

\section{Case 5}

A 60 year old left-handed retired policeman had developed progressive personality and behavioural change over a 6 year period, beginning with the acute onset of paranoid and persecutory delusions. $\mathrm{He}$ believed that colleagues were plotting to poison him and breaking into his house. When challenged he offered an elaborate defense of these beliefs and could not be dissuaded from holding them. He took an overdose of paracetamol and was admitted to hospital for treatment of depression. There was some response to antidepressant therapy and he was able to return to work briefly before taking early retirement. His social skills further deteriorated, and he behaved aggressively toward strangers and family members. He developed a sweet tooth, and began smoking and drinking heavily. He became increasingly obsessed with purchasing bargain items, spending much time seeking these. Subsequently he became withdrawn and apathetic, and would lie in bed for days at a time. A further trial of antidepressant therapy (sertraline) produced no benefit. There was a past medical history of ischaemic heart disease, hypertension and impaired glucose tolerance, but no history of psychiatric illness. Medications at the time of the onset of the neruobehavioural syndrome comprised aspirin, atenolol and simvastatin. His mother had developed behavioural change and paranoia in her fifties prior to her death from a stroke. 
On examination, he was distractible and impassive with a fatuous demeanour, and adynamic aphasia. Executive deficits were evident. The general neurological examination was normal.

\section{Case 6}

A 53 year old male policeman presented with progressive personality and behavioural change over a three-year period. Work colleagues complained about his poor performance and social skills and his attendance became erratic. He became withdrawn and self-absorbed, though there were no associated mood symptoms. Within the first year of the illness, he began describing detailed delusions involving famous football players. He believed that one of these football players regularly shopped at his local supermarket, and that he had seen him there on many occasions. He would also tell his wife that he had received large sums of money or gifts of odd items such as electrical appliances and when challenged, would offer plausible explanations. He developed a sweet tooth, hoarding of food and other obsessional behaviours and routines, and would frequently talk about religion. $\mathrm{He}$ neglected self-care and required prompting to change his clothes, and he had increasing difficulty performing simple tasks such as using the cooker. Spontaneous speech became impoverished. There was a past history of depression five years previously, which responded successfully to antidepressant therapy (citalopram). His father had been hospitalised with depression and was found to have been stealing prior to his death from cancer aged 56.

On examination, the patient was impulsive and perseverative, with a flat affect and poor attention. Spontaneous speech was impoverished but with normal articulation, prosody and grammar. There was evidence of executive impairment and mild word retrieval difficulty. The general neurological examination was normal.

\section{Case 7}

A 55 year old woman had developed progressive personality change and impairment of language over a period of 3 years. She became increasingly disinhibited and childlike, resulting in the loss of her job as a playground supervisor. Early in the course of the disease, she developed grandiose delusions. She believed that others thought she was a well-known television personality, and this led her to approach strangers in public and remonstrate with them. She developed an intense dislike of elderly people. She became increasingly preoccupied with home security and engaged in prolonged checking and tidying rituals. She also refused to handle post, which she believed was contaminated, and would repeatedly wash her hands. She insisted on painting household objects certain colours. She became obsessed with the appearance of her nose, and repeatedly requested corrective surgery. She became increasingly withdrawn and less interested in certain activities such as reading which she had previously enjoyed; however she would listen avidly to a small repertoire of classical music. These behavioural problems were accompanied by progressive difficulties in word-finding, spelling and recognition of familiar faces. The past medical history included hypertension, but no history of psychiatric illness; she took no medications. Her father had been in a nursing home for many years with dementia attributed to chronic alcoholism.

On examination, she was disinhibited and fatuous. Speech was fluent with occasional paraphasic errors, and there was evidence of impaired single word comprehension, surface dyslexia and executive dysfunction. There was orofacial apraxia, a positive pout and bilateral grasp reflexes.

\section{Case 8}

An 84 year old woman presented with progressive cognitive and neuropsychiatric deficits over five years. Her first symptom was wordfinding difficulty and subsequently comprehension of language deteriorated, with evolution of a typical SD syndrome. Her memory for events and places appeared largely intact. Early in the illness she de- veloped a delusion of infestation. She attributed benign physical features such as veins and bunions to worms. She reported that she could feel the worms crawling under her skin, causing a prickling sensation over her whole body and also felt them moving within the sinuses of her face. She also believed that her abdomen had become swollen. She was convinced that her cognitive problems were caused by the worm infestation, and would not be persuaded otherwise. These symptoms were accompanied by loss of empathy and rigidity of thought. There was no past medical or psychiatric history and no family history of neurological illness.

On examination, speech was fluent with anomia and frequent circumlocutions, and there was surface dyslexia. The general neurological examination was normal.

\section{Discussion}

Here we have described a series of eight patients with prominent delusions in the context of FTLD. The diagnosis of FTLD was confirmed pathologically in two cases (and in the parent of a third case from an autosomal dominant pedigree). There was suspicion of a relevant family history in at least one other case (Case 5). All patients in this series fulfilled consensus clinical criteria for FTLD $[1,2]$ and additionally had supportive neuropsychology and typical brain MRI findings (Table 1). These cases demonstrated radiological features previously identified as hallmarks of FTLD [2-4]: regional atrophy predominantly involving the frontal and/or anterior temporal lobes with asymmetric involvement of the cerebral hemispheres. Behavioural variant FTLD was the most frequent clinical subtype, though syndromes of tvFTLD (SD with left temporal lobe atrophy in Case 8) and FTD-MND (pathologically confirmed in Case 1) were also represented. In contrast, none of our patients had the syndrome of PNFA. None of the patients had evidence for an alternative, non-degenerative condition likely to have contributed to the development of delusions; there was a previous episode of depression in Case 6, but this had resolved with antidepressant therapy several years before the onset of the behavioural syndrome. Case 1 had received corticosteroid therapy but this is unlikely to have been the cause of delusions: therapy predated the onset of the behavioural syndrome by a number of years and symptoms began on a reduced dosage regimen. None of our patients had clinical features suggestive of $\mathrm{AD}$ (early global episodic memory impairment, topographical disorientation) or DLB (extrapyramidal signs, fluctuation), or radiological evidence of significant vascular disease. Case 8 , however, is somewhat problematic: while this patient fulfilled consensus criteria for the SD syndrome, the advanced age at onset here does raise the possibility of a focal variant of $\mathrm{AD}$. While the SD syndrome is unusual as a presentation of $\mathrm{AD}$ [15], the presence of atypical features (as here) may increase the likelihood of AD pathology.

The estimated prevalence of delusions in FTLD cohorts in previous studies has ranged from $0 \%$ to $23 \%$ 
[10-12, 16-18]. The estimated prevalence of delusions in patients presenting with FTLD in our tertiary referral population was approximately $14 \%(8 / 56)$. This compares with the variable but overall substantially greater prevalence (approximately 17-70\%) in AD and DLB, estimated from large series $[10,19-20]$, though such estimates do not take into account the relative disease stage at which delusions appear. However, it is difficult to estimate the true prevalence of delusions in FTLD based on a retrospective series, as here. Retrospectively acquired cases are subject to ascertainment bias, which tends to over-estimate prevalence. On the other hand, a history of delusions may not have been volunteered spontaneously by patients or carers, particularly as other behavioural and cognitive deficits supervened.

As the present study was retrospective, it was not possible to analyse the characteristics or syndromic associations of delusions uniformly in the entire cohort of FTLD patients. However, delusions were an early and prominent feature of disease, occurring as a leading symptom within a year of clinical onset in seven of the eight patients included in this series. In some cases, delusions preceded the development of cognitive deficits, and bore no obvious relationship to other measures of disease duration or severity (Table 1). Delusions were an enduring feature of FTLD in this series, persisting between two and seven years from onset to the time of recording. There may be a distinction here from other degenerative disorders such as AD or DLB, in which persistent significant delusions tend to occur as a later feature in the context of established neurological disease [20]. Delusional ideation early in the course of $\mathrm{AD}$ often involves themes of theft and may be attributable to loss of insight into the individual's own memory difficulties [22]; none of our patients had delusions of this kind. Within the FTLD spectrum, the bvFTLD syndrome and subcortical pathologies (FTD-MND and corticobasal degeneration) appear to be over-represented $[18,21]$. Histopathologically, ubiquitin-positive, tau-negative neuronal inclusions (with or without evidence of motor neuron disease) have been most frequently associated with delusions in FTLD [18, 21, 23, 24]. It remains possible that delusions in patients with features of FTLD signal an alternative pathology in at least a proportion of cases, and this can only be resolved by case series with pathological confirmation.

Little information is available concerning the detailed phenomenology of delusions in FTLD, their relationship to other neuropsychiatric and cognitive features, or whether they resemble delusions in other neurological and psychiatric disorders. In one previous study of a patient with pathologically confirmed Pick's disease, religious and paranoid delusions were accompanied by auditory hallucinations [25]. In another study, three patients with pathologically confirmed FTD-MND presented with positive psychotic symptoms: one had a presentation similar to our Case 1, delusions preceding motor symptoms by several years [21]. Bak et al. [23] described a patient with pathologically confirmed FTDMND who presented with delusions of a phantom lodger similar to Case 1 here. Another patient with FTLD and pathologically confirmed ubiquitin-positive, tau-negative pathology at post mortem presented with prominent delusions of erotomania, somewhat similar to our Case 2 [24]. The delusions described by our patients were phenomenologically rich and diverse (Table 1 ), including paranoid and persecutory delusions, delusions involving famous people, somatic delusions and delusions of parasitosis/infestation and body part distortion. The psychotic symptoms in our cases were resistant to treatment. It is of interest that certain cardinal symptoms of schizophrenia, such as thought interference, passivity and third-person auditory hallucinations, were not represented, while on the other hand, two patients developed tactile hallucinations (a rarer symptom in schizophrenia). However, the characteristics of the delusions exhibited by our patients are consistent with current formulations that emphasise the often fantastic nature and personal significance of delusional content (for example, Scharfetter's definition of a delusion as 'an overriding rigid conviction which creates a self-evident, private, and isolating reality requiring no proof' [26]).

From a clinical standpoint, the present series raises the possibility that FTLD may account for an unrecognized proportion of cases of late-life delusions. Some of our cases were initially misdiagnosed as having treatment-resistant depression, while patients with late-onset paraphrenia present with psychotic symptoms at a similar age (in contrast to classical schizophrenia, which tends to affect a younger population). Asymmetrical temporal lobe atrophy, a cardinal feature of FTLD, has been described in individuals with paraphrenia [27] and in patients with cognitive impairment associated with delusions [12,22]: such patients may represent undiagnosed cases of FTLD. Taken together, these observations suggest that further investigations, including brain imaging, are required in any patient with late onset, refractory psychiatric illness and associated behavioural or cognitive deficits, particularly if these evolve over time. Conversely, in patients presenting with features suggestive of FTLD, delusions and other neuropsychiatric symptoms should be actively sought, as they may not be volunteered spontaneously but may contribute to the early decline in inter-personal conduct that is a cardinal feature of FTLD.

The case numbers in this study were small, the findings are based on retrospective analysis of a tertiary referral population and pathological confirmation was obtained in only a minority. Prospective studies are needed to establish whether our findings generalise to the wider population of patients with FTLD in the community, and in particular to determine the true preva- 
lence of delusions in FTLD versus alternative pathologies. Further work will be required to investigate the pathophysiological, histopathological and anatomical correlates of delusions and other neuropsychiatric symptoms in FTLD, to evaluate their clinical and prognostic significance, and to establish their place in differentiating FTLD from other neurodegenerative and psychiatric disorders (or indeed, the proportion of cases in which delusions may signal an alternative pathology). This may be particularly timely as the diagnostic criteria for bvFTLD continue to be critically redefined [28].
Acknowledgements We thank Dr John Stevens for assistance in analysis of brain images; Dr Miriam Prins, Dr Rene Stolwyk, Ms Natasja Van Harskamp, Dr Karen Oaksford, Ms Gail Robinson, Dr Antonio Incisa della Rocchetta and Ms Julia Hailstone for assistance with neuropsychological assessments; and Dr Janice Holton, Prof Tamas Revesz and Prof Peter Lantos for performing the neuropathological examinations. This work was undertaken at UCLH/UCL who received a proportion of funding from the Department of Health's NIHR Biomedical Research Centres funding scheme. This work is supported by the UK Alzheimer Research Trust and Medical Research Council. JDW is supported by a Wellcome Trust Intermediate Clinical Fellowship. CTL was the recipient of an Alzheimer's Australia Research Travelling Scholarship. The Dementia Research Centre is an Alzheimer's Research Trust Coordinating Centre.

Conflict of interest The authors declare no conflict of interest.

\section{References}

1. Neary D, Snowden JS, Gustafson L, Passant U, Stuss D, Black S, Freedman M, Kertesz A, Robert PH, Albert M, Boone K, Miller BL, Cummings J, Benson DF (1998) Frontotemporal lobar degeneration: a consensus on clinical diagnostic criteria. Neurology 51:1546-1554

2. McKhann GM, Albert MS, Grossman M, Miller B, Dickson D, Trojanowski JQ (2001) Clinical and pathological diagnosis of frontotemporal dementia. Arch Neurol 58:1803-1809

3. Chan D, Fox NC, Scahill RI, Crum WR, Whitwell JL, Leschziner G, Rossor AM, Stevens JM, Cipolotti L, Rossor MN (2001) Patterns of temporal lobe atrophy in semantic dementia and Alzheimer's disease. Ann Neurol 49:433-442

4. Rosen HJ, Gorno-Tempini ML, Goldman WP, Perry RJ, Schuff N, Weiner M, Feiwell R, Kramer JH, Miller BL (2002) Patterns of brain atrophy in frontotemporal dementia and semantic dementia. Neurology 58:198-208

5. Ogar JM, Dronkers NF, Brambati SM, Miller BL, Gorno-Tempini ML (2007) Progressive nonfluent aphasia and its characteristic motor speech deficits. Alzheimer Dis Assoc Disord 21: S23-S30

6. Cairns NJ, Bigio EH, Mackenzie IRA, et al. (2007) Neuropathologic diagnostic and nosologic criteria for frontotemporal lobar degeneration: consensus of the Consortium for Frontotemporal Lobar Degeneration. Acta Neuropathologica 114:5-22

7. Gregory CA (1999) Frontal variant of frontotemporal dementia: a crosssectional and longitudinal study of neuropsychiatric features. Psychol Med 29:1205-1217
8. Swartz JR, Miller BL, Lesser IM, Booth R, Darby A, Wohl M, Benson DF (1997) Behavioral phenomenology in $\mathrm{Alz}$ heimer's disease, frontotemporal dementia, and late-life depression: a retrospective analysis. J Geriatr Psychiatry Neurol 10:67-74

9. Gregory CA, McKenna PJ, Hodges JR (1998) Dementia of frontal type and simple schizophrenia: two sides of the same coin? Neurocase 4:1-6

10. Mendez MF, Shapira JS, Woods RJ, Licht EA, Saul RE (2008) Psychotic symptoms in frontotemporal dementia: prevalence and review. Dement Geriatr Cogn Disord 25:206-211

11. Levy ML, Miller BL, Cummings JL, Fairbanks LA, Craig A (1996) Alzheimer disease and frontotemporal dementias. Behavioral distinctions. Arch Neurol 53:687-690

12. Engelborghs S, Maertens K, Nagels G, Vloeberghs E, Marien P, Symons A, Ketels V, Estercam S, Somers N, De Deyn PP (2005) Neuropsychiatric symptoms of dementia: cross-sectional analysis from a prospective, longitudinal Belgian study. Int J Geriatr Psychiatry 20:1028-1037

13. Arciniegas DB, Topkoff JL, Held K, Frey L (2001) Psychosis due to neurologic conditions. Curr Treat.Options Neurol 3:347-366

14. Diagnostic and Statistical Manual of Mental Disorders, $4^{\text {th }}$ Edition (1994) American Psychiatric Publishing: Arlington

15. Alladi S, Xuereb J, Bak T, Nestor P, Knibb J, Patterson K, Hodges JR (2007) Focal cortical presentations of Alzheimer's disease. Brain 130:2636-2645

16. Hirono N, Mori E, Tanimukai S, Kazui $\mathrm{H}$, Hashimoto M, Hanihara T, Imamura $\mathrm{T}$ (1999) Distinctive neurobehavioral features among neurodegenerative dementias. J Neuropsychiatry Clin Neurosci 11:498-503
17. Mendez MF, McMurtray A, Chen AK, Shapira JS, Mishkin F, Miller BL (2006) Functional neuroimaging and presenting psychiatric features in frontotemporal dementia. J Neurol Neurosurg Psychiatry 77:4-7

18. Hodges JR, Davies RR, Xuereb JH, Casey B, Broe M, Bak TH, Kril JJ, Halliday GM (2004) Clinicopathological correlates in frontotemporal dementia. Ann Neurol 56:399-406

19. Ballard CG, O'Brien JT, Swann AG, Thompson P, Neill D, McKeith IG (2001) The natural history of psychosis and depression in dementia with Lewy bodies and Alzheimer's disease: persistence and new cases over 1 year of follow-up. J Clin Psychiatry 62:46-49

20. Scarmeas N, Brandt J, Albert M, Hadjigeorgiou G, Papadimitriou A, Dubois B, Sarazin M, Devanand D, Honig L, Marder K, Bell K, Wegesin D, Blacker D, Stern Y (2005) Delusions and hallucinations are associated with worse outcome in Alzheimer disease. Arch Neurol 62:1601-1608

21. Nitrini R, Rosemberg S (1998) Psychotic symptoms in dementia associated with motor neuron disease: a pathophysiological hypothesis. J Neuropsychiatry Clin Neurosci 10:456-458

22. Geroldi C, Akkawi NM, Galluzzi S, Ubezio M, Binetti G, Zanetti O, Trabucchi M, Frisoni GB (2000) Temporal lobe asymmetry in patients with Alzheimer's disease with delusions. J Neurol Neurosurg Psychiatry 69: 187-191

23. Bak TH, O'Donovan DG, Xuereb JH, Boniface S, Hodges JR (2001) Selective impairment of verb processing associated with pathological changes in Brodmann areas 44 and 45 in the motor neurone disease-dementia-aphasia syndrome. Brain 124:103-120 
24. Tartaglia MC, Kertesz A, Ang LC (2008) Delusions and hallucinations in frontotemporal dementia: a clinicopathologic case report. Cogn Behav Neurol 21:107-110

25. Waddington JL, Youssef HA, Farrell MA, Toland J (1995) Initial 'schizophrenia-like' psychosis in Pick's disease: case study with neuroimaging and neuropathology, and implications for frontotemporal dysfunction in schizophrenia. Schizophr Res 18:79-82
26. Scharfetter C (1980) General psychopathology: An introduction. transl. Marshall H. Cambridge, Cambridge University Press

27. Howard R, Mellers J, Petty R, Bonner D, Menon R, Almeida O, Graves M, Renshaw C, Levy R (1995) Magnetic resonance imaging volumetric measurements of the superior temporal gyrus, hippocampus, parahippocampal gyrus, frontal and temporal lobes in late paraphrenia. Psychol Med 25:495-503
28. Rascovsky K, Hodges JR, Kipps CM, et al. (2007) Diagnostic criteria for the behavioral variant of frontotemporal dementia (bvFTD): current limitations and future directions. Alzheimer Dis Assoc Disord 21:S14-S18 